\title{
Newton's Bucket Experiment from Kantian Perspective
}

\author{
Yaohua Zhu \\ Nankai University, Tianjin, China \\ Email: zhuyaohua1992@163.com
}

How to cite this paper: Zhu, Y. H. (2018). Newton's Bucket Experiment from Kantian Perspective. Open Journal of Philosophy, 8 , 437-443.

https://doi.org/10.4236/ojpp.2018.85030

Received: July 23, 2018

Accepted: September 15, 2018

Published: September 18, 2018

Copyright $\odot 2018$ by author and Scientific Research Publishing Inc. This work is licensed under the Creative Commons Attribution International License (CC BY 4.0).

http://creativecommons.org/licenses/by/4.0/

\begin{abstract}
Newton's absolute pace-time view is the basis of his classical mechanics, and his description of relative motion is based on absolute space. However, the existence of this absolute space has been questioned by the academic circles. In order to defend its theoretical foundation, Newton established a famous bucket experiment to prove the existence of absolute space. But his experiment was also questioned, and the experiment is also divided into different opinions. This paper hopes to find the answer to the question from Kantian perspective.
\end{abstract}

\section{Keywords}

Absolute Space, Bucket Experiment, Motion, Kant, Idea

\section{Introduction}

In The Mathematical Principles of Natural Philosophy, Newton said, "absolute space, as far as its nature is concerned, has nothing to do with the outside world, but it's always the same stationary" (Newton, 1957). Newton believes that this absolute space is the absolute stationary reference frame, and that there are other relative reference frames that move uniformly and in a straight line being relative to it (Maciel \& Tiomno, 1989). In order to prove the existence of the absolute stationary reference frame (absolute space), Newton established the famous experiment which called bucket experiment.

The design concept of bucket experiment is that if we can observe a relatively stationary object showing a state of motion, then we can think that the motion of this object is not relative, but absolute (Newton, 1934). Because according to the law of inertia, we can only observe that the object is moving or just in a stationary state in a relative inertial reference frame. So, this absolute motion does not 
depend on the current relative frame of reference, but rather a motion relative to an absolute space.

However, Kant put forward the opposite view.

In the metaphysical principle of natural science, Kant pointed out, "Absolute space is therefore necessary, not as a concept of an actual object, but rather as an idea, which is to serve as a rule for considering all motion therein merely as relative; and all motion and rest must be reduced to absolute space, if the appearance thereof is to be transformed into a determinate concept of experience (which unites all appearances)" (Kant, 2004). But Kant thought that absolute space is not an empty space from the phoronomical sense, but only the idea of a space. In this, we exclude all particular matter that makes it known as the empirical object, so that the material space or any other empirical space is still conceived as motion in it. Thus, movement is not only conceived as absolute, but always regarded as only a relative predicate in the mutual relation (Watkins, 1997).

On this basis, Kant denied the existence of absolute motion and absolute space. Kant thought that "all motion or rest can be relative only and never absolute, that is, that matter can be thought as moved or at rest solely in relation to matter, and never with respect to mere space without matter, so that absolute motion, thought without any relation of one matter to another, is completely impossible" (Kant, 2004).

Therefore, from Kantian point of view, Newton's bucket experiment must also be incorrect. Thus, it is necessary to analyze Newton's bucket experiment to explain whether absolute motion and absolute space exist.

\section{The Bucket Experiment}

The rotating bucket experiment is a thought experiment set up by Newton. I will briefly divided the experimental process into four steps as follows.

\subsection{The Process of Experiment}

At first, hanging the bucket on a rope. Rotating barrel gradually until the rope is tight, and then with the appropriate amount of water into the bucket, both the bucket and water are keeping static. The surface of water is flat now in this first step. In the second step, when the bucket is released, it will begin to spin gradually because of the tight rope. At the beginning of the bucket rotating, the water in the bucket has not rotated yet, and the surface of the water is still flat. After a period of time, the water rotates with the friction between the bucket and water, and the surface of water begins to form a concave, which is the third step. Until the rotation rate of water is consistent with the bucket, they are relatively stationary. Water is static relative to the bucket in the last step. The surface of water is not flat, but is still concave. It can be find in the (Laymon, 1978).

The important point of this experiment is when the bucket and water are relatively stationary in step 1 and step 4 , the state of the water's surface is different (In Step 1 is flat, but in Step 4 is concave). 


\subsection{The Explanation of Newton}

Newton made an explanation of the two different situations. He thought the rise of water shows the tendency of moving away from the axis of rotation, which shows water is doing absolute circular motion. This movement can be observed directly. At the beginning, the surface of the water is flat, so its circular motion has not really begun. At this point, the relative movement of the water in the bucket is greatest, but there is no tendency to leave the shaft. But later, the water in the bucket gradually began to rotate, and the relative motion gradually decreased. Water began to move toward the edge of bucket, which proved that it had a tendency to leave the rotating shaft. This tendency indicates that the true circle motion of water is increasing until it reaches its maximum value, when the water is relatively static in the bucket (Maudlin, 1993).

In other words, there are two cases which make water relative to the bucket keeping stationary: The first is neither of them were rotating. That is the surface of the water was flat only when neither of them were rotating. The second is both of them were rotating. That is the surface of the water was curved, when both of them were rotating synchronously.

As follows, Newton made a deductive reasoning.

There are five premise in this reasoning.

1) There are two relative static states of water and bucket.

2) There are two states of water surface: flat and concave.

3) When the bucket does not rotate, the surface of the water is flat.

4) When the bucket rotates, the surface of the water is concave.

5) The concave surface of the water shows its tendency to move away from the shaft, indicating that the water is moving.

Through these five premises, we can get two conclusions. The first one is the motion of water does not depend on its relative motion, it is independent and absolute motion. The other is the motion tendency of water depends on an absolute reference frame-absolute space.

\subsection{Problems in Reasoning}

At first, all the premise of 1) - 4) can be directly observed, but the 5th premise are different from them, because it contains some components of inference.

In the forth step of bucket experiment (bucket and water are relatively static, the surface of water is concave), the movement of water's surface can't be perceived, when the bucket is used as the reference system. So the perception of surface motion should be a inference. Thus the basis for such inference should be at least three reference frames (There is a third reference frames besides water and bucket), because the inference that the concave surface is still moving has used a background knowledge of Newton's law of inertia.

However, on the basis of this inertial theory, if we have to infer that water is still moving relative to the reference frame of the bucket, then it can only be concluded that its motion is relative to another reference frame. So before that, 
we need to know how we form an understanding of the motion of an object, and whether this knowledge of the motion of an object is reliable by inference (Johansson, 1995). Based on it, we can know that, in the bucket experiment, does this another reference frame belong to the absolute space or still belong to the relative inertial reference frame?

\section{From Kantian Point of View}

Kant has put forward his original view on our understanding of motion from epistemology. He thought that "matter is the movable insofar as it, as such a thing, can be an object of experience. For its representation to become experience, we require, in addition, that something be thought through the understanding" (Kant, 1999). What's more, motion is change of relation in space. There are thus always two correlates here (Pollok, 2006).

In bucket experiment, there are three objects that stimulate our senses, which were bucket, water, and entities that provide gravity. At the same time, there is a correlation among them each other. Because the gravity is the main source of centripetal force in the experiment. If there is no gravity, the water does not appear this kind of change, and will not be able to provide us with the sensory stimulation that water is flat in the first step. So it is a necessary condition for the existence of the experiment.

In step 1 of the experiment (bucket and water are relative stationary, and the surface of water is flat), we can draw the knowledge that the water surface is relatively stationary, but actually the relative static between the three elements which are bucket, water and the entities that provide gravity.

In step 4 of the experiment (bucket and water are relative stationary, the surface of water is concave), we still get the knowledge of the relative stillness of the water surface, and we still get the knowledge that the water surface is relatively stationary.

The surface of water has changed from step 4 to step 3 in this experiment $[\Delta(4 \rightarrow 3)]$, Newton concluded that the water was moving.

The formation of this phenomenon $\Delta(4 \rightarrow 3)$ actually stemmed from three objects (bucket, water and the entities that provide gravity) that stimulate our senses at the same time. Therefore, the perceptual judgments we make about relative movements are produced by the contrast among these three elements. Without the stimulation of the entity that generates gravity, we can not form knowledge in step 1 about the relative stillness of water and bucket. Therefore, this is a triangular relation among bucket, water and entities that provide gravity. When bucket and water are relatively stationary, the judgment of the whole motion state of them depends entirely on the reference to the entities that provide gravity. So, Newton's absolute motion of water through $\Delta(4 \rightarrow 3)$ is actually relative motion relative to the entities that provide gravity.

Obviously, if the entities that provide gravity is absolute, then the motion judgment that $\Delta(4 \rightarrow 3)$ gets is absolute motion. But the entities that provide 
gravity is transcendent existence for us, the phenomenon in this experiment is derived from these three thing-in-themselves. How can we obtain the knowledge of them?

If there is an absolute space entity, the relative space eventually end in the absolute one. Then we need many times to carry the experiment of bucket, constantly looking for another entity which produces the phenomenon of $\Delta(4 \rightarrow 3)$. As long as all is exhausted, we can find the absolute space entity. But the number of entities in the universe is limited or not is a rational knowledge, which is about what the universe itself is. It doesn't belong to the phenomenal world, it is the domain of thing-in-itself, and belongs to transcendent existence, it can only serve as an idea. If it serves as knowledge, it becomes a rational phantasm. In this case, how should we treat the concept of absolute space?

\section{Absolute Space Exists as a Idea}

Just as Kant said that "motion is change of relation in space. There are thus always two correlates here" (Kant, 1999). However, the absolute space that in contrast to relative (empirical) space is not the object of experience, so it is impossible for absolute motion without reference to anything empirical. It can be seen from Kant's phenomenological point of view, if we want to provide motion as a appearance, we must need an empirical representation of space so that the object of motion can change its relationship with it, but the space that should be perceived must be material (Kant, 1997). When we continue to think of this space as motion, we can think of it as being contained within a space of greater extent, and take the latter to be at rest. "But the same can be done with the latter, with respect to a still further extended space, and so on to infinity, without ever arriving by experience at an immovable (immaterial) space, with respect to which either motion or rest might absolutely be attributed to any matter" (Kant, 1999). Thus, all movement or stillness is relative, not absolute. That is to say, there is no movement or stillness that is divorced from the relation to matter. So, this absolute motion that does not depend on any relation of one matter to another is impossible.

But Kant believed that this did not mean that the concept of absolute space should not exist, we can imagine it as an idea which should be used as a rule for considering all motion therein merely as relative, rather than a concept of an actual object. And if we want to transform all motion or static phenomena into a determinate concept of experience (which unifies all appearances), it must be reduced to absolute space.

\section{Conclusions}

To sum up, the bucket experiment didn't prove the existence of absolute space, and the absolute motion obtained by Newton through this experiment was still the relative motion of water, which was relative to the entities that provided gravity. 
For the concept of absolute space, Kant believed that the reason is that they are divorced from the experience of evidence, and there is no evidence to prove its limitation and infinite. They both have no inspection standard, so no matter what they say, there's no way to make a decision. So you should hold onto the realm of phenomena to describe a knowledge, and don't refer to the unknowable domain of thing-in-itself. The debate about the domain of thing-in-itself is beyond the scope of experience, and it is all wrong to say yes or no. Thus, in the bucket experiment, the tendency of water surface from $\Delta(4 \rightarrow 3)$ does not prove that it is moving relative to the absolute space.

Although the existence of absolute space can't be confirmed and can't be denied, we can still have such a rational belief that its value in practice is undeniable. In Newton's mechanics theory, it is precisely because of the introduction of the concept of absolute space that Newton's law of motion based on inertial system is established (Marinov, 1979). This is also the significance of the concept of absolute space.

\section{Conflicts of Interest}

The author declares no conflicts of interest regarding the publication of this paper.

\section{References}

Johansson, G. (1995). Visual Motion Perception. Scientific American, 92, 2433. http://psycnet.apa.org/psycinfo/1975-28753-001

Kant, I. (1997). Prolegomena to any Future Metaphysics. Journal of Philosophy, 48, 507. https://www.questia.com/read/55086895/prolegomena-to-any-future-metaphysics

Kant, I. (1999). Critique of Pure Reason (pp. 208-220). Cambridge, UK: Cambridge University Press.

Kant, I. (2004). Metaphysical Foundations of Natural Science. Cambridge, UK: Cambridge University Press.

Laymon, R. (1978). Newton's Bucket Experiment. Journal of the History of Philosophy, 16, 399-413. http://muse.jhu.edu/article/227468/pdf https://doi.org/10.1353/hph.2008.0681

Maciel, A. K. A., \& Tiomno, J. (1989). Analysis of Absolute Space-time Lorentz Theories. Foundations of Physics, 19, 505-519. https://link.springer.com/article/10.1007/BF00734656 https://doi.org/10.1007/BF00734656

Marinov, S. (1979). The Coordinate Transformations of the Absolute Space-Time Theory. Foundations of Physics, 9, 445-460. https://link.springer.com/article/10.1007/BF00708535 https://doi.org/10.1007/BF00708535

Maudlin, T. (1993). Buckets of Water and Waves of Space: Why Spacetime Is Probably a Substance. Philosophy of Science, 60, 183-203.

http://www.mendeley.com/catalog/buckets-water-waves-space-spacetime-probably-su bstance/

https://doi.org/10.1086/289728 
Newton, I. S. (1934). Sir Isaac Newton's Mathematical Principles of Natural Philosophy and His System of the World. California: University of California Press.

Newton, I. S. (1957). The Mathematical Principles of Natural Philosophy (pp. 208-220). Beijing: Commercial Press.

Pollok, K. (2006). Kant's Critical Concepts of Motion. Journal of the History of Philosophy, 44, 559-575.

https://www.researchgate.net/publication/236715527_Kant's_Critical_Concepts_of_M otion https://doi.org/10.1353/hph.2006.0071

Watkins, E. (1997). The Laws of Motion from Newton to Kant. Perspectives on Science, 5, 14P-15P.

http://www.researchgate.net/publication/267107458_The_laws_of_motion_from_Newt on_to_Kant 